\title{
Investigation into the Origin of Age-Velocity dispersion Relation by High Resolution Simulation
}

\author{
Jun Kumamoto ${ }^{1,2}$, Junichi Baba ${ }^{2}$ and Takayuki R. Saitoh ${ }^{2}$ \\ ${ }^{1}$ Astronomical Institute, Tohoku University, \\ Aramaki, Aoba, Sendai, Miyagi 980-8578, Japan. \\ ${ }^{2}$ Earth-Life Science Institute, Tokyo Institute of Technology, \\ Ookayama, Meguro, Tokyo 152-8551, Japan.
}

\begin{abstract}
We focused on the stellar age-velocity dispersion relation (AVR). We performed the N-body/SPH simulations to investigate the origin of AVR. As a results, we found that AVR is not consistent with simple stellar heating.
\end{abstract}

Keywords. Galaxy: kinematics and dynamics, methods: numerical

\section{Introduction}

We focus on the stellar age-velocity dispersion relation (AVR). Observation of the AVR in the vicinity of the Sun shows that older stars have larger velocity dispersions (Holmberg et al. 2009). This trend indicates that stars may be heated by giant molecular clouds (GMCs), spiral arms or minor mergers. However, details of AVR are not understood. Some researchers suggested that gravitational scattering by GMCs is too weak to reproduce observed AVR (e.g., Hänninen \& Flynn 2002). However, these previous numerical work include simple model without calculating gas dynamics, star formation, and so on.

\section{Overview}

We performed high resolution three-dimensional N-body/SPH simulations with ASURA (Saitoh et al. 2008) to investigate the origin of AVR. In our simulation, we assumed a static halo and disk, the profiles of which are respectively described by the NavarroFrenk-White (NFW) profile and the Miyamoto-Nagai model. A gaseous disk is simple exponential profile with 1 million SPH particles. Our models include star formation, radiative cooling, and supernovae feedback. As a result, we found that AVR is not consistent with simple stellar heating. The gaseous state vary with time, and so the strength of gravitational scattering by GMCs is different from moments to moments. This result suggests that GMCs can be heating sources to establish AVR observed in the vicinity of the Sun in spite of weak heating.

\section{References}

Hänninen, J. \& Flynn, C. 2002, MNRAS, 337, 731

Holmberg, J., Nordström, B., \& Andersen, J. 2009, A\&A, 501, 941

Saitoh, T. R., Daisaka, H., Kokubo, E., Makino, J., Okamoto, T., Tomisaka, K., Wada, K., \& Yoshida, N. 2008, PASJ, 60, 667 